\title{
Amateurs' Contribution to the PHEMU85 Campaign Observing Mutual Phenomena of Jupiter's Satellites
}

\author{
J.E. Arlot \\ Bureau des Longitudes, 77 avenue Denfert-Rochereau, \\ F-75014 Paris, France
}

Mutual events (occultations or eclipses) of Jupiter's satellites occur every 6 years. The 1985 series was particularly favourable as it occurred when Jupiter was at opposition. An observing campaign (PHEMU85) was therefore organized by the Bureau des Longitudes, and sponsored by the CNRS under RCP (Coordinated Research Programme) 754.

The interest in observing the events lies in the precise positional information that may be obtained. Conventional (photographic) astrometry gives results to within $400 \mathrm{~km}$, but mutual events give an accuracy of at least $100 \mathrm{~km}$. Observation of mutual events is easy in that the Galilean satellites are bright, so large telescopes do not have to be used, and photoelectric observations in particular can be obtained from urban sites or under poor meteorological conditions. The professional workers generally employed photoelectric photometers or vidicon-type detectors, while amateurs used visual, photographic, photometric and video techniques. Two groups of amateurs were particularly involved, GEOS (European) and GEA (Spain), but individual amateurs also contributed.

The observations essentially consist of recording the magnitudes over the duration of the events (about 10 minutes), and the time (UTC). Although photoelectric observations gave the highest precision, some visual light-curves have sufficient accuracy for them to be used in future theoretical studies. Occultations, where the amplitude is small, were difficult to observe visually, but were detected.

Photographic observations used standard 35-mm film (Ilford HPS), a 30-cm telescope and 4-second exposures, and obtained scientifically interesting results.

We hope to mount a similar campaign in 1991 and invite amateurs interested in participating to contact us as soon as possible. 